\title{
Design of Small CRPA Arrays with Circular Microstrip Loops for Electromagnetically Coupled Feed
}

\author{
Jun Hur ${ }^{1} \cdot$ Gangil Byun ${ }^{2, *} \cdot$ Hosung Choo $^{1}$
}

\begin{abstract}
This paper proposes a design of small controlled reception pattern antenna (CRPA) arrays using circular microstrip loops with frequencyinsensitive characteristics. The proposed array consists of seven identical upper and lower circular loops that are electromagnetically coupled, which results in a frequency-insensitive behavior. To demonstrate the feasibility of the proposed feeding mechanism, the proposed array is fabricated, and its antenna characteristics are measured in a full-anechoic chamber. The operating principle of the proposed feeding mechanism is then interpreted using an equivalent circuit model, and the effectiveness of the circular loop shape is demonstrated by calculating near electromagnetic fields in proximity to the radiator. The results confirm that the proposed feeding mechanism is suitable to have frequency-insensitive behavior and induces strong electric and magnetic field strengths for higher radiation gain in extremely small antenna arrays.
\end{abstract}

Key Words: Controlled Reception Pattern Antenna, Electromagnetically Coupled Feed, Frequency-Insensitive Characteristic, Microstrip Loop Antenna.

\section{INTRODUCTION}

Recent technical issues on controlled reception pattern antenna (CRPA) arrays are mainly focused on the employment of more antenna elements in an electrically restricted area [1-4]. However, the increasing number of antenna elements causes mutual coupling that degrades the impedance matching characteristics and radiation efficiency due to undesired shifts in the resonance frequencies $[5,6]$. In addition, a typical rectangular patch antenna has frequency-sensitive characteristics due to its narrow matching bandwidth with an extremely steep reactance slope. Thus, a lot of efforts have been made to broaden the matching bandwidth by using additional parasitic elements, via pins, and external matching circuits [7-9]; however, these approaches in- crease the design complexity and require an additional layer to implement the extra structure. Although Kang et al. [10] proposed the design of rectangular microstrip loop antennas for a frequency-insensitive behavior with lower complexity, there has not been an in-depth consideration for reducing mutual coupling effects to further improve the antenna characteristics in extremely restricted areas.

In this paper, we propose the design of small CRPA arrays using circular microstrip loops with an electromagnetically coupled feed. The proposed array is composed of seven individual elements printed on high-dielectric ceramic substrates, and each element has upper and lower circular loops that are designed to resonate at $1.575 \mathrm{GHz}$ and $1.227 \mathrm{GHz}$, respectively. The lower loop is fed by two output ports of an external hybrid chip coupler

Manuscript received January 7, 2018 ; Revised March 14, 2018 ; Accepted April 4, 2018. (ID No. 20180107-004J)

${ }^{1}$ Department of Electronic and Electrical Engineering, Hongik University, Seoul, Korea.

${ }^{2}$ Department of Electrical and Computer Engineering, Ulsan National Institute of Science and Technology (UNIST), Ulsan, Korea.

"Corresponding Author: Gangil Byun (email: byun@unist.ac.kr)

This is an Open-Access article distributed under the terms of the Creative Commons Attribution Non-Commercial License (http://creativecommons.org/licenses/by-nc/4.0) which permit unrestricted non-commercial use, distribution, and reproduction in any medium, provided the original work is properly cited.

(c) Copyright The Korean Institute of Electromagnetic Engineering and Science. All Rights Reserved. 
(XC1400P-03S; Anaren Inc., Syracuse, NY, USA) embedded on a printed circuit board $(\mathrm{PCB})$ at the bottom of the array ground. The lower loop is fed by two output ports of an external hybrid chip coupler embedded on a PCB at the bottom of the array ground. The upper loop is then electromagnetically coupled by the near-fields radiated from the lower loop, and this feeding mechanism allows for frequency-insensitive behavior with lower reactance slope. Another advantage of this feeding structure is that the ratio between the electric and magnetic coupling strengths can be independently adjusted by varying the substrate thickness and the loop widths. Note that the tuning capability is essential for maximizing the antenna characteristics in small arrays by compensating for extra capacitance and inductance, which come from the strong mutual coupling. To verify the feasibility of the proposed feeding mechanism with circular microstrip loops, the seven-element array is fabricated, and its antenna characteristics are measured in a full-anechoic chamber. The operating principle of the proposed feeding mechanism is interpreted by building an equivalent circuit model, and the near electromagnetic fields are also observed to analyze the effectiveness of the circular loop shape. The results demonstrate that the proposed feeding mechanism is suitable to maintain a lower reactance slope and higher radiation gain in extremely small antenna arrays.

\section{Proposed CiRCULAR LOOP ANTENNA ARRAY}

Fig. 1 shows the geometry of the proposed seven-element CRPA array. The array has a circular ground with a radius of $r$, and its array elements are placed at a distance of $m$ from the center of the ground. Each element is composed of upper and lower circular loops that are printed on high-dielectric ceramic substrates $\left(\varepsilon_{r}=20, \tan \delta=0.0035\right)$ with thicknesses of $h_{1}$ and $h_{2}$. The upper and lower circular loops of each element are designed with diameters of $l_{1}$ and $l_{2}$ and widths of $w_{1}$ and $w_{2}$. The lower loop is directly fed by two output ports of an external hybrid chip coupler embedded on the PCB, and the upper loop is electromagnetically coupled to the lower loop. The circumferences of the upper and lower loops are then tuned for their resonate frequencies of 1.575 $\mathrm{GHz}$ and $1.227 \mathrm{GHz}$, respectively. Note that $h_{1}$, $w_{1}$, and $w_{2}$ are important design parameters of the proposed feeding mechanism to adjust the ratio between the electric and magnetic coupling strengths for improved matching and radiation properties. The detailed design parameters are optimized by a genetic algorithm in conjunction with the FEKO EM simulator (Altair, Detroit, MI, USA) [11], and the optimized values are listed in Table 1.

Fig. 2(a) presents a photograph of the proposed array printed on the ceramic substrate. Fig. 2(b) shows the PCB that includes

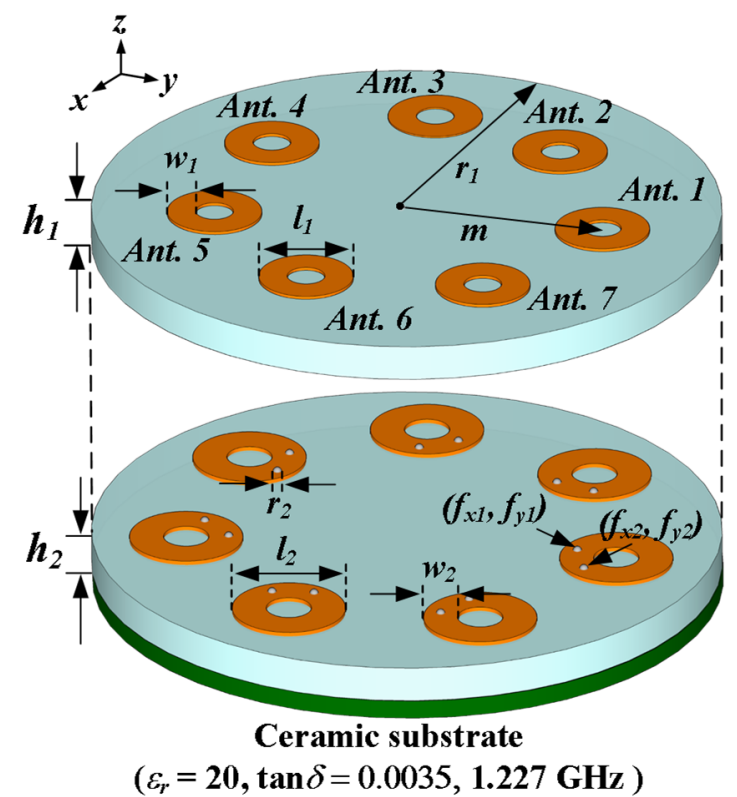

(a)

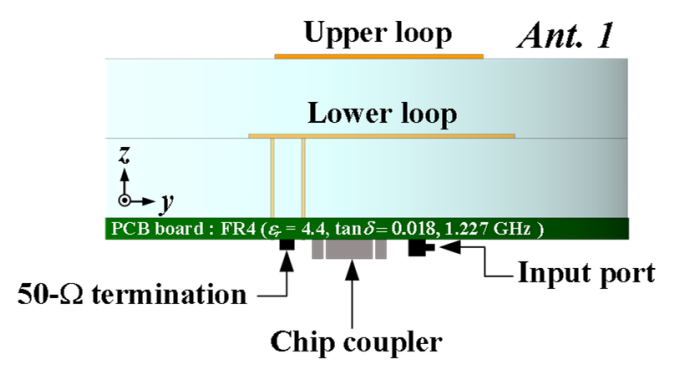

(b)

Fig. 1. Geometry of the proposed array: (a) perspective view and (b) side view of the Ant.1.

the hybrid chip coupler with coplanar wave guides and $50-\Omega$ termination chips for quadrature phase excitation. The antenna characteristics such as the reflection coefficient and active element pattern are measured in a full anechoic chamber, and the results will be discussed in Section III.

Table 1. Optimized values of the proposed array

\begin{tabular}{cc}
\hline Parameter & Value \\
\hline$r_{1}$ & $63.5 \mathrm{~mm}$ \\
$r_{2}$ & $0.8 \mathrm{~mm}$ \\
$m$ & $45 \mathrm{~mm}$ \\
$h_{1}$ & $6 \mathrm{~mm}$ \\
$h_{2}$ & $6 \mathrm{~mm}$ \\
$l_{1}$ & $19.3 \mathrm{~mm}$ \\
$l_{2}$ & $21.1 \mathrm{~mm}$ \\
$w_{1}$ & $3.25 \mathrm{~mm}$ \\
$w_{2}$ & $5.6 \mathrm{~mm}$ \\
$\left(f_{x 1}, f_{y 1}\right)$ & $(-37.9,12.2)$ \\
$\left(f_{x 2}, f_{y 2}\right)$ & $(-33.2,22)$ \\
\hline
\end{tabular}




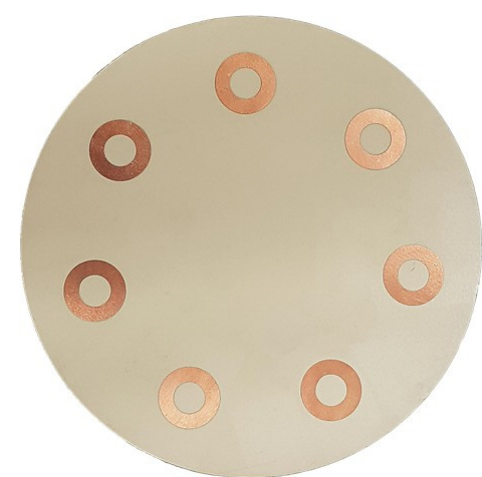

(a)

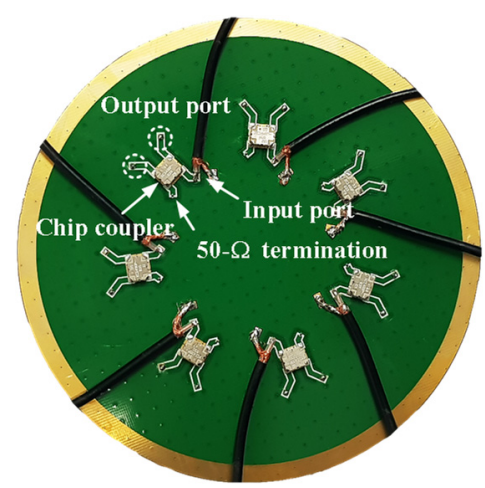

(b)

Fig. 2. Photographs of the fabricated array: (a) top view and (b) bottom view.

\section{MEASUREMENT AND ANALYSIS}

Fig. 3(a) shows the simulated reflection coefficients, specified by a dashed line, in comparison with measured results of the Ant.1. The results are obtained when Ant.1 is fed by a coaxial probe, while the other antennas are terminated by $50-\Omega$ loads. The measured values are $-17.71 \mathrm{~dB}$ and $-24.76 \mathrm{~dB}$ at $1.575 \mathrm{GHz}$ and $1.227 \mathrm{GHz}$, respectively. The simulated values are $-17.71 \mathrm{~dB}$ and $-24.76 \mathrm{~dB}$ at $1.575 \mathrm{GHz}$ and $1.227 \mathrm{GHz}$, respectively. As can be seen, the simulated and measured reflection coefficients show similar tendency and maintain values less than $-10 \mathrm{~dB}$ over a wide frequency range from $1.1 \mathrm{GHz}$ to $1.7 \mathrm{GHz}$. This broadband impedance matching performance caused not only by the antenna characteristic, but also by the hybrid chip coupler where the reflected power at the antenna input port comes to the isolated port of the coupler in the operating frequency. Fig. 3(b) presents the mutual coupling between Ant.1, Ant.2, Ant.3, and Ant.4. Other cases are omitted because they have the same separation distances. The simulated and measured coupling strengths have similar tendency and less than $-18 \mathrm{~dB}$ and $-10 \mathrm{~dB}$ at 1.575 $\mathrm{GHz}$ and $1.227 \mathrm{GHz}$. Fig. 4 represents a comparison between the simulated and measured bore-sight gains of the Ant.1. The measured and simulated values are $-3.2 \mathrm{dBic}$ and $-1.9 \mathrm{dBic}$ at $1.575 \mathrm{GHz}$ and $-5.1 \mathrm{dBic}$ and $-4.6 \mathrm{dBic}$ at $1.227 \mathrm{GHz}$.

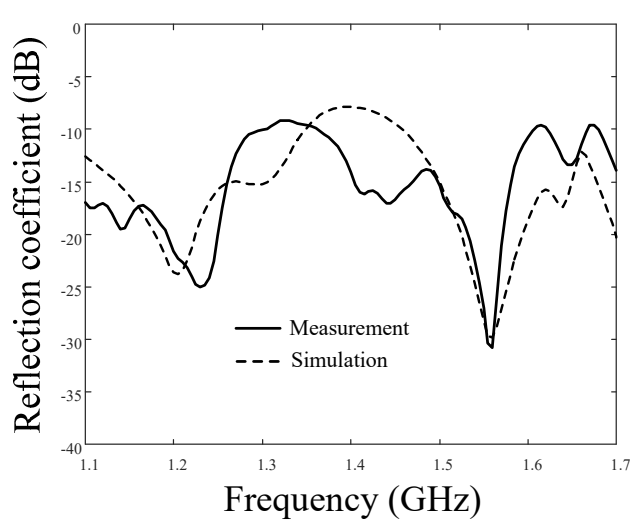

(a)

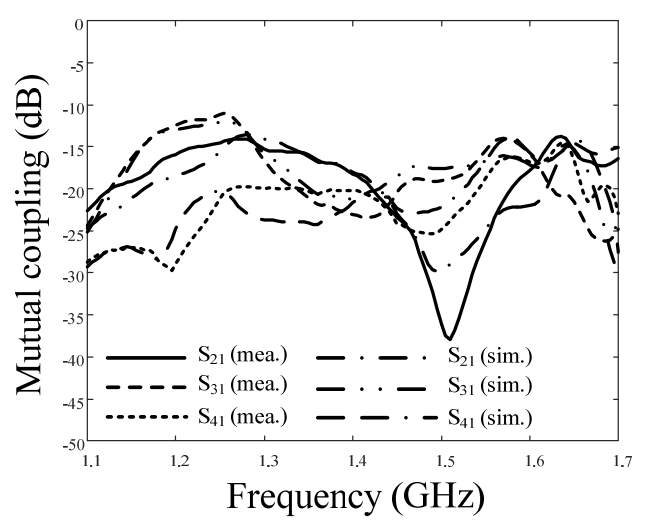

(b)

Fig. 3. $S$-parameters of the proposed array: (a) reflection coefficient and (b) mutual coupling.

The simulated antenna efficiencies for single antenna element without adjacent elements are $76 \%(1.575 \mathrm{GHz})$ and $83 \%$ (1.227 $\mathrm{GHz}$ ), while the antenna efficiencies with adjacent array elements are $33 \%(1.575 \mathrm{GHz})$ and $28 \%(1.227 \mathrm{GHz})$. The gain deviation from $1.15 \mathrm{GHz}$ to $1.65 \mathrm{GHz}$ is less than $10 \mathrm{~dB}$, which is lower than a typical patch antenna which uses only electric or magnetic coupled feeding mechanism. These results demonstrate that the proposed electromagnetically coupled feed structure can derive the frequency-insensitive behavior.

Fig. 5 provides an axial ratio of Ant.1 in the bore-sight direc-

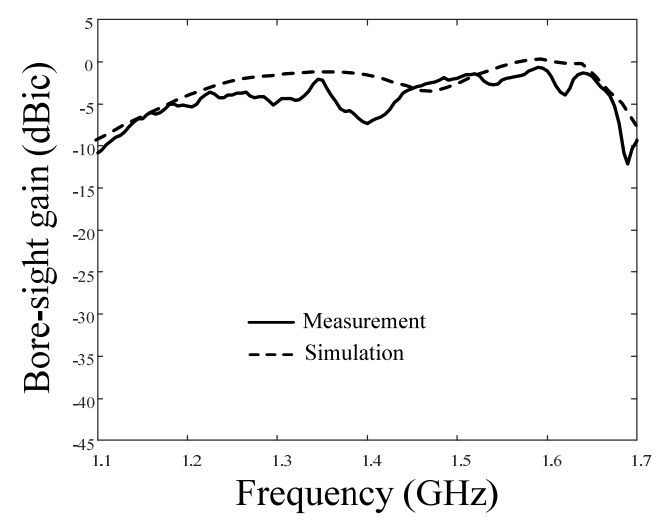

Fig. 4. Bore-sight gain of the proposed array (Ant.1). 


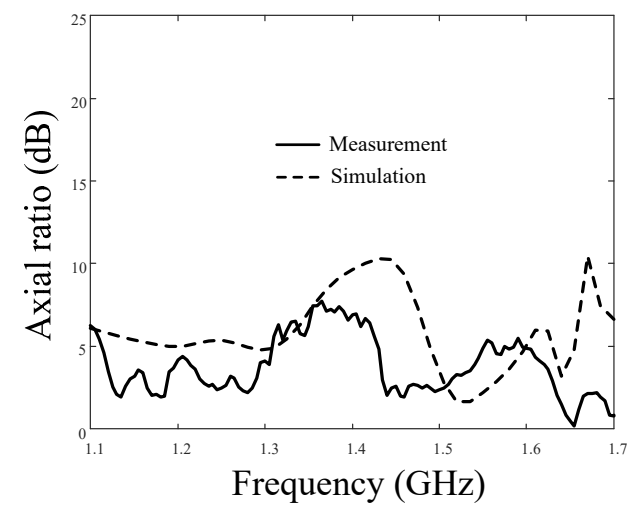

Fig. 5. Axial ratio of the proposed array (Ant.1).

tion. The antenna has simulated $\mathrm{AR}$ values of $2.8 \mathrm{~dB}$ and $5.1 \mathrm{~dB}$ at $1.575 \mathrm{GHz}$ and $1.227 \mathrm{GHz}$, respectively, and the measured values are $3.8 \mathrm{~dB}$ at $1.575 \mathrm{GHz}$ and $3.6 \mathrm{~dB}$ at $1.227 \mathrm{GHz}$. The $A R$ values within the half-power beamwidth (HPBW) are listed in Table 2. The radiation patterns of Ant.1 in the $z x$ - and $z y$ planes at GPS L1/L2 bands are shown in Fig. 6. The measured HPBWs at $1.575 \mathrm{GHz}$ are $130^{\circ}$ in the $z x$-plane and $115^{\circ}$ in the $z y$-plane. The HPBWs at $1.227 \mathrm{GHz}$ have values of $90^{\circ}$ and $120^{\circ}$ in the $z x$ - and $z y$-planes, respectively. As expected, the antenna does not exhibit any serious pattern distortion in the upper hemisphere with HPBWs of greater than $90^{\circ}$, which is suitable for use in the CRPA applications.

To interpret the operating principle of the proposed antenna from a circuit point of view, an equivalent circuit model is built using a data-fitting method, as shown in Fig. 7(a). The circuit describes the input impedance at port 1 , while terminating the port 2 with $50-\Omega$ impedance. The via pin connected to the lower loop is represented as inductance $L_{F}$, and another parallel circuit, composed of $R_{P}, L_{P}$, and $C_{P}$, is inserted to describe the higherorder resonance characteristic above $2 \mathrm{GHz}$. The lower loop is composed of $R_{L 2}, L_{L 2}$, and $C_{L 2}$, and the upper loop is specified by $R_{L 1}, L_{L 1}$, and $C_{L 1}$. These lower and upper loops are linked through the series capacitance $C_{c}$ and the coupling coefficient $k$, which are associated with the electric and magnetic couplings, respectively. Note that the frequency-insensitive reactance variations can be obtained by employing the optimal ratio of $C_{c}$ and $k$. The detailed values of the lumped elements are listed in Table 3, and the input impedance of the circuit is compared with that of the antenna obtained from the FEKO EM simulator, as illustrated in Fig. 7(b).

To verify the advantage of the proposed feeding mechanism, we compare reactance variations with other feeding mechanisms using only electric or magnetic couplings, as shown in Fig 8 . The dashed line represents the feeding mechanism that uses only the electric coupling after removing the inductive coupling coefficient $k$. The dotted line indicates the response when the capacitance $C_{c}$ is removed. The results demonstrate that the propo-
Table 2. Axial ratio values of the proposed array (Ant.1)

\begin{tabular}{ccc}
\hline$(\theta, \phi)$ & $\begin{array}{c}\text { Measurement } \\
(\mathrm{L} 1, \mathrm{~L} 2)\end{array}$ & $\begin{array}{c}\text { Simulation } \\
(\mathrm{L} 1, \mathrm{~L} 2)\end{array}$ \\
\hline$\left(0^{\circ}, 0^{\circ}\right)$ & $(2.8,5.1)$ & $(3.8,3.6)$ \\
$\left(20^{\circ}, 0^{\circ}\right)$ & $(2.5,5.2)$ & $(2.6,4.7)$ \\
$\left(40^{\circ}, 0^{\circ}\right)$ & $(1.8,4.9)$ & $(2.1,4.4)$ \\
$\left(60^{\circ}, 0^{\circ}\right)$ & $(3.1,5.6)$ & $(3.2,4.9)$ \\
$\left(80^{\circ}, 0^{\circ}\right)$ & $(7.6,7.9)$ & $(6.7,7.5)$ \\
$\left(-20^{\circ}, 90^{\circ}\right)$ & $(2.6,4.3)$ & $(3.2,4.1)$ \\
$\left(-40^{\circ}, 90^{\circ}\right)$ & $(2.9,6.3)$ & $(3.2,5.6)$ \\
$\left(-60^{\circ}, 90^{\circ}\right)$ & $(3.8,10.6)$ & $(3.9,9.0)$ \\
$\left(-80^{\circ}, 90^{\circ}\right)$ & $(5.8,18.6)$ & $(6.0,16.9)$ \\
\hline
\end{tabular}

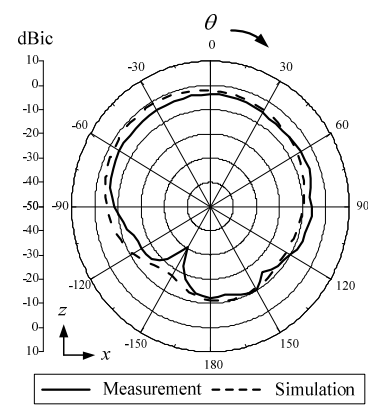

(a)

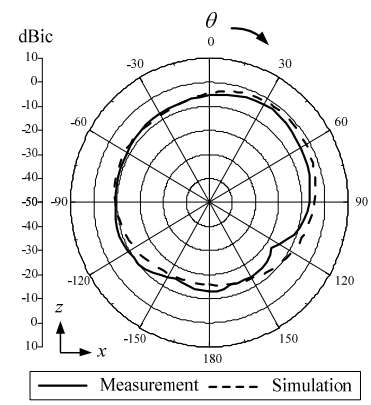

(c)

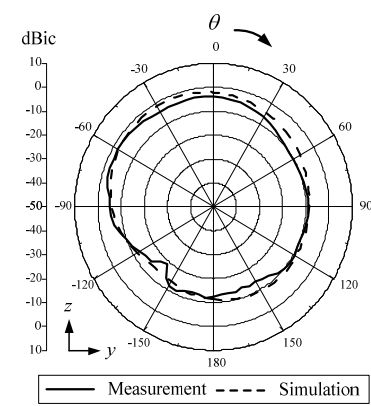

(b)

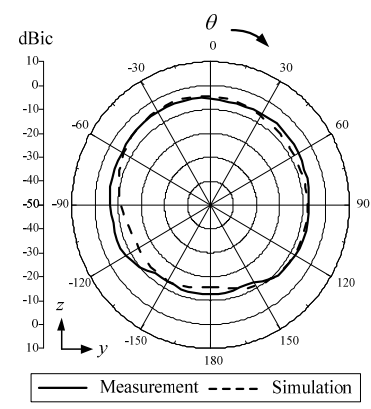

(d)
Fig. 6. Radiation patterns of the proposed array (Ant.1): (a) $z x$-plane at $1.575 \mathrm{GHz}$, (b) $z y$-plane at $1.575 \mathrm{GHz}$, (c) $z x$-plane at 1.227 $\mathrm{GHz}$, and (d) zy-plane at $1.227 \mathrm{GHz}$.

sed electromagnetic coupling with the solid line has a frequencyinsensitive reactance variation with lower reactance slope compared to other feeding mechanisms.

Fig. 9 shows the average electric and magnetic field distributions at $1.575 \mathrm{GHz}$ and $1.227 \mathrm{GHz}$ according to the shape of the loops. For a fair comparison, we optimized the circular and rectangular loops to resonate at same frequencies $(1.575 \mathrm{GHz}$, $1.227 \mathrm{GHz})$ under the identical substrate diameter $\left(r_{1}=30 \mathrm{~mm}\right)$ and thickness $\left(h_{1}=h_{2}=6 \mathrm{~mm}\right.$ ). Fig. 9(a) presents a comparison of electric field distributions induced below the outer edge of the upper loop as specified by the dotted lines in the inset figure. The average field strengths are calculated according to the observation heights, and the results confirm that the circular loop maintains consistently higher field intensity. For example, the maximum 


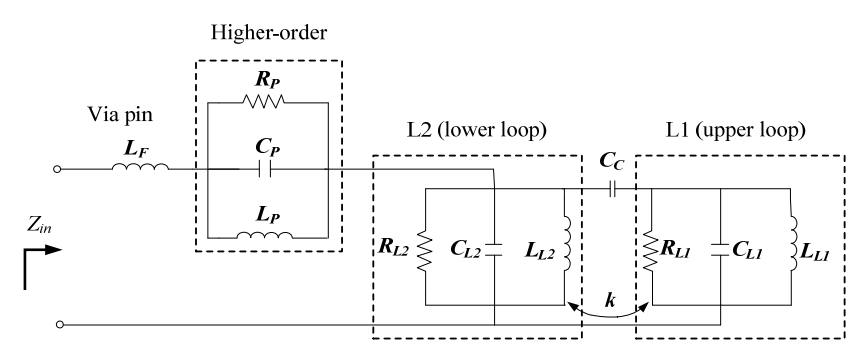

(a)

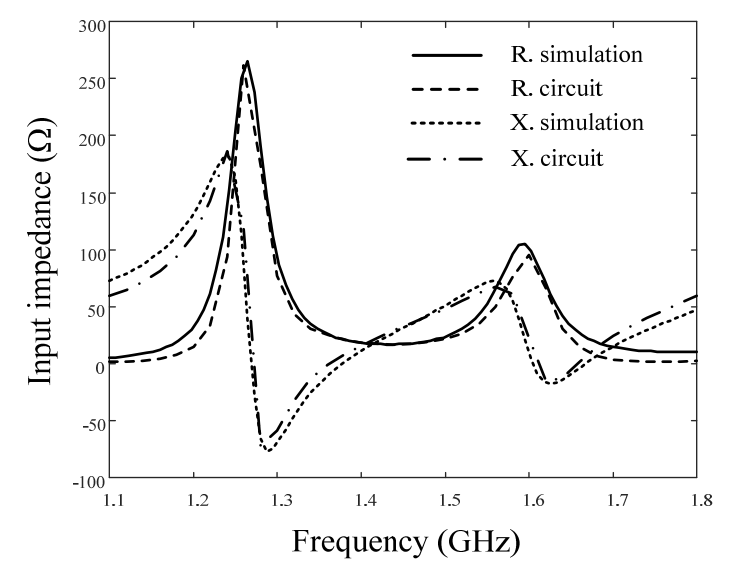

(b)

Fig. 7. Equivalent circuit model and its input impedance: (a) circuit model and (b) input impedance.

difference is found at $11 \mathrm{~mm}$ with a value of $2.5 \mathrm{~dB}$, which helps to achieve stronger electric coupling in the feeding mechanism. We also observed the magnetic field distributions induced at the highlighted area of the inset figure in Fig. 9(b). As similarly observed in the electric field distributions, the circular shape of the radiator helps to induce a stronger magnetic flux compared to the rectangular loop shape, which is required to achieve frequencyinsensitive behavior in such extremely small arrays. The anti-jamming characteristic can be observed by applying nulling algorithms such as the power inversion (PI) and least mean square (LMS), to the proposed antenna $[12,13]$.

Table 3. Values of the lumped elements

\begin{tabular}{cc}
\hline Parameter & Value \\
\hline$L_{F}$ & $2.1 \mathrm{nH}$ \\
$L_{P}$ & $1.6 \mathrm{nH}$ \\
$C_{P}$ & $3.4 \mathrm{pF}$ \\
$R_{P}$ & $443 \Omega$ \\
$R_{L 2}$ & $600 \Omega$ \\
$L_{L 2}$ & $1.8 \mathrm{nH}$ \\
$C_{L 2}$ & $7.2 \mathrm{pF}$ \\
$C_{c}$ & $0.5 \mathrm{pF}$ \\
$k$ & 0.2 \\
$R_{L 1}$ & $250 \Omega$ \\
$L_{L 1}$ & $1.7 \mathrm{nH}$ \\
$C_{L 1}$ & $6.6 \mathrm{pF}$ \\
\hline
\end{tabular}

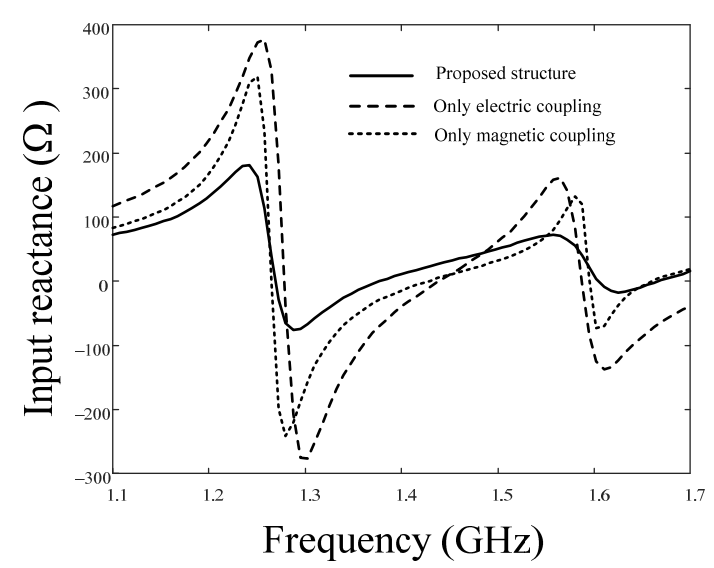

Fig. 8. Reactance variations according to the feeding mechanism.

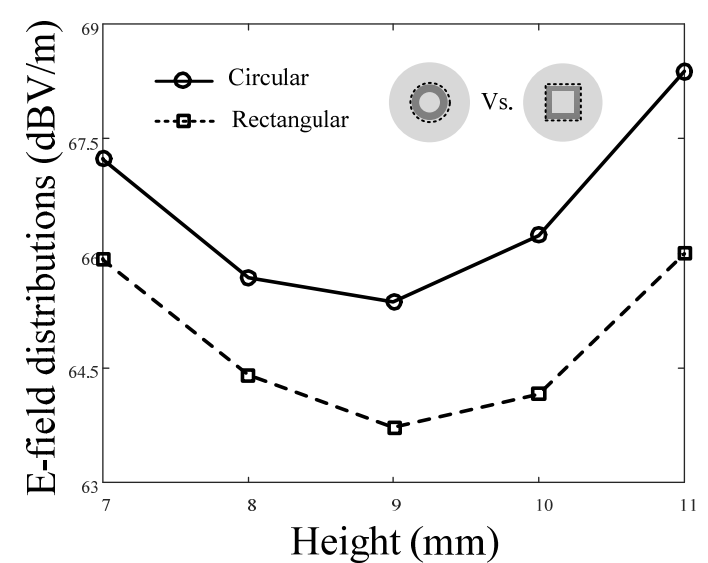

(a)

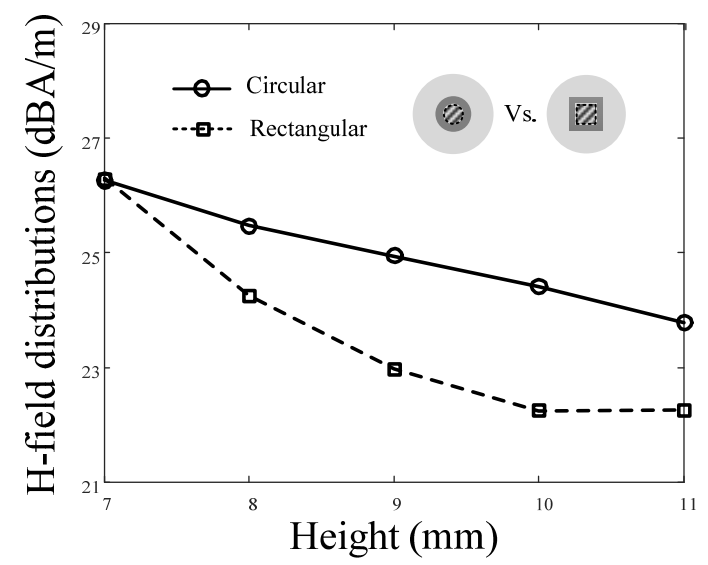

(b)

Fig. 9. Average field distributions according to the shape of the loop: (a) E-field at edge of the loop, (b) H-field inside of the loop.

\section{CONCLUSION}

We investigated the design of small CRPA arrays using the electromagnetically coupled feed with circular microstrip loops. The lower loop was fed by the external hybrid chip coupler, and the upper loop was electromagnetically coupled to the lower loop, which allowed for the frequency-insensitive behavior with the broadband circular polarization. The fabricated array showed 
measured reflection coefficients of $-17.71 \mathrm{~dB}$ and $-24.76 \mathrm{~dB}$ with bore-sight gains of $-3.2 \mathrm{dBic}$ and $-5.1 \mathrm{dBic}$ at $1.575 \mathrm{GHz}$ and $1.227 \mathrm{GHz}$, respectively. In addition, the gain deviation between $1.15 \mathrm{GHz}$ to $1.65 \mathrm{GHz}$ was less than $10 \mathrm{~dB}$. We also observed the near electromagnetic fields in proximity to the antenna and verified that the circular loop shape was capable of inducing stronger fields with the maximum improvement of $5.1 \mathrm{~dB}$ (electric field) and $7.0 \mathrm{~dB}$ (magnetic field). The results demonstrate that the proposed feeding mechanism is suitable to achieve the frequency-insensitive behavior with the lower reactance slope in the presence of strong mutual coupling.

This work was supported by the National Research Foundation of Korea (NRF) grant funded by the Korea government (MSIP) (No. NRF-2017R1D1A1B04031890) and in part by the Basic Science Research Program through the NRF funded by the Ministry of Education (No. 2015R1A6A1A03031833).

\section{REFERENCES}

[1] R. L. Fante and J. J. Vaccaro, "Wideband cancellation of interference in a GPS receive array," IEEE Transactions on Aerospace and Electronic Systems, vol. 36, no. 2, pp. 549-564, 2000.

[2] G. Byun, H. Choo, and S. Kim, "Improvement of pattern null depth and width using a curved array with two subarrays for CRPA systems," IEEE Transactions on Antennas and Propagation, vol. 63, no. 6, pp. 2824-2827, 2015.

[3] G. Byun, J. C. Hyun, S. M. Seo, and H. Choo, "Optimum array configuration to improve null steering time for mobile CRPA systems," Journal of Electromagnetic Engineering and Science, vol. 16, no. 2, pp. 74-79, 2016.

[4] S. G. Ha, J. Cho, and K. Y. Jung, "Design of miniaturized microstrip patch antennas using non-Foster circuits for compact controlled reception pattern antenna array," Journal of Electromagnetic Engineering and Science, vol. 17, no. 2, pp.
108-110, 2017.

[5] Y. Zhang and H. Zhao, "Failure diagnosis of a uniform linear array in the presence of mutual coupling," IEEE Antennas and Wireless Propagation Letters, vol. 14, pp. 1010-1013, 2015.

[6] H. J. Song, A. Bekaryan, J. H. Schaffner, A. Hussain, and P. S. Kildar, "Effects of mutual coupling on LTE MIMO capacity for monopole array: comparing reverberation chamber tests and drive tests," IEEE Antennas and Wireless Propagation Letters, vol. 14, pp. 454-457, 2014.

[7] A. Kaya and E. Y. Yuksel, "Investigation of a compensated rectangular microstrip antenna with negative capacitor and negative inductor for bandwidth enhancement," IEEE Trans. Antennas Propag., vol. 55, no. 5, pp. 1275-1282, May 2007.

[8] Y. X. Guo, L. Bian, and X. Q. Shi, "Broadband circularly polarized annular-ring microstrip antenna," IEEE Transactions on Antennas and Propagation, vol. 57, no. 8, pp. 24742477, 2009.

[9] S. Fu, Q. Kong, S. Fang, and Z. Wang, "Broadband circularly polarized microstrip antenna with coplanar parasitic ring slot patch for L-band satellite system application," IEEE Antennas and Wireless Propagation Letters, vol. 13, pp. 943-946, 2014.

[10] M. C. Kang, H. Choo, and G. Byun, "Design of a dualband microstrip loop antenna with frequency-insensitive reactance variations for an extremely small array," IEEE Transactions on Antennas and Propagation, vol. 65, no. 6, pp. 2865-2873, 2017.

[11] Y. Rahmat-Samii and E. Michielssen, Electromagnetic Optimization by Genetic Algorithms. Hoboken, NJ: Wiley, 1999.

[12] R. T. Compton, "The power-inversion adaptive array: concept and performance," IEEE Transactions on Aerospace and Electronic Systems, vol. 15, no. 6, pp. 803-814, 1979.

[13] O. L. Frost, "An algorithm for linearly constrained adaptive array processing," Proceedings of the IEEE, vol. 60, no. 8, pp. 926-935, 1972. 
Jun Hur

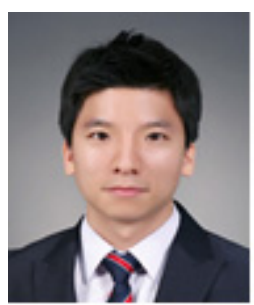

forming. received the B.S. and M.S. degrees in electronic and electrical engineering from Hongik University, Seoul, Korea, in 2014 and 2016, respectively. He is currently working toward the Ph.D. degree in electronics and computer engineering at Hongik University, Seoul, Korea. His research interests include the global positioning system antennas, antenna arrays, and position optimization of array elements for adaptive beam-

\section{Gangil Byun}

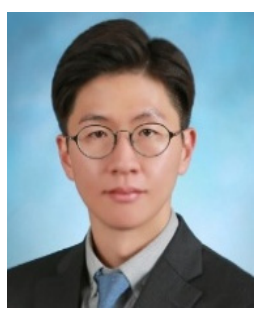

received his B.S. and M.S. degrees in electronic and electrical engineering from Hongik University, Seoul, Korea in 2010 and 2012, respectively, and his Ph.D. degree in electronics and computer engineering from Hanyang University, Seoul, Korea in 2015. He joined the faculty of Ulsan National Institute of Science and Technology (UNIST) in February 2018 and is currently Assistant Professor of Electrical and Computer Engineering (ECE). Dr. Byun's principal areas of research are in the design and analysis of small antenna arrays for adaptive beamforming applications, such as direction of arrival estimation, interference mitigation, and radar. His recent research interests also include circularly-polarized antennas, vehicular and aeronautic antennas, global positioning system antennas, antenna and array configuration optimization.

\section{Hosung Choo}

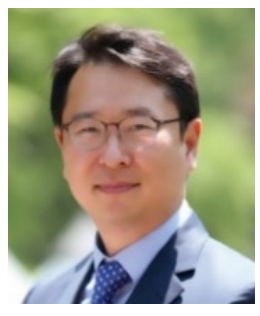

received the B.S. degree in radio science and engineering from Hanyang University in Seoul in 1998, and the M.S. and Ph.D. degrees in electrical and computer engineering from the University of Texas at Austin, in 2000 and 2003, respectively. In September 2003, he joined the School of Electronic and Electrical Engineering, Hongik University, Seoul, Korea, where he is currently a full professor. His principal areas of research are the use of the optimization algorithm in developing antennas and microwave absorbers. His studies include the design of small antennas for wireless communications, reader and tag antennas for RFID, and on-glass and conformal antennas for vehicles and aircraft. 